\title{
Nitrogen-doped Diamond Nanowire Gas Sensor for the Detection of Methane
}

\author{
Andrew F. Zhou ${ }^{1, *}$, Xinpeng Wang ${ }^{2}$, Peter Feng ${ }^{3, *}$ \\ ${ }^{1}$ Department of Physics, Indiana University of Pennsylvania, Indiana, PA 15705, USA \\ ${ }^{2}$ Nanonex Corporation, Monmouth Junction, New Jersey 08852, USA \\ ${ }^{3}$ Department of Physics, University of Puerto Rico, San Juan, Puerto Rico 00936, USA \\ *Corresponding authors: E-mail: fzhou@iup.edu; peter.feng@upr.edu
}

DOI: $10.5185 /$ amlett.2020.021474

Diamond-based sensors have shown great potential in the past few years due to their unique physicochemical properties. We report on the development of high-performance nitrogen-doped ultrananocrystalline diamond (UNCD) nanowire-based methane $\left(\mathrm{CH}_{4}\right)$ gas sensors, taking advantage of a large surface-to-volume ratio and a small active area offered by the 1D nanowire geometry. The morphologic surface and crystalline structures of UNCD are also characterized by using scanning electron microscopy (SEM) and Raman scattering, respectively. By using synthesized nanowire arrays combined with 4-pin electrical electrodes, prototypic highly sensitive $\mathrm{CH}_{4}$ gas sensors have been designed, fabricated and tested. Various parameters including the sensitivity, response and recovery times, and thermal effect on the performance of the gas sensor have also been investigated in order to quantitate the sensing ability. Enhanced by the small grain size and porosity of the nanowire structure, fabricated nanowire UNCD sensors demonstrated a high sensitivity to $\mathrm{CH}_{4}$ gas at room temperature down to 2 ppm, as well as fast response and recovery times which are almost 10 times faster than that of regular nanodiamond thin film based sensors.

\section{Introduction}

Various diamond-based gas sensors have been demonstrated to detect hydrogen, oxygen, carbon monoxide, etc., based on the surface conductivity change with adsorbed gas molecules [1-3]. Diamond based gas sensors are expected to have high sensitivity, fast response, and good tolerance to high temperatures. For example, X. Peng et al. reported on boron-doped ultrananocrystalline diamond (UNCD) nanowire based carbon monoxide (CO) gas sensors, [4] while Yasar Gurbuz et al. built borondoped polycrystalline diamond (PCD) film based sensors for the detection of benzene $\left(\mathrm{C}_{6} \mathrm{H}_{6}\right)$ and toluene $\left(\mathrm{C}_{7} \mathrm{H}_{8}\right)$ [5]. Hydrogenated nanocrystalline diamond (NCD) films synthesized using chemical vapor deposition (CVD) techniques have been demonstrated for the detection of ammonia $\left(\mathrm{NH}_{3}\right)$ [6]. The three-dimensional model of the current density distribution of the hydrogenated NCD was used to describe the transient flow of electrons between interdigitated electrodes and the hydrogenated NCD surface [7].

It is difficult to make nanowires based sensing devices on NCD because of its rough surface. Therefore, UNCD nanowire array has been used in the present work. Besides some of the important characteristics including high stability and chemical inertness, the UNCD films have excellent thickness uniformity with surface roughness of 4$7 \mathrm{~nm}$ which does not change with an increase of the UNCD film thickness $[\mathbf{8 , 9}]$.
Recently, a $4.8 \%$ sensitivity at 500 ppm has been reported for a lithium doped carbon nanotubes $(\mathrm{CNT}) \mathrm{CH}_{4}$ sensor at room temperature [10]. The current studies have shown progress in the development of diamond film and nanostructure-based gas sensors. However, very few papers report on gas sensors based on nitrogen-doped (N-doped) diamond materials, especially with nanowire geometry. It will be interesting to see if a nanowire diamond-based methane gas sensor could offer better performance, although we observed the electrical property change of nitrogen-doped ultrananocrystalline diamond caused by methane gas [11].

In this paper, we present experimental results on the fabrication of N-doped UNCD nanowire arrays for gas sensor applications. Both crystalline structures and electrical properties of UNCD nanowire arrays have been characterized. The focus of our experimental studies is on the basic properties of the nitrogen-doped UNCD nanowire $\mathrm{CH}_{4}$ gas sensor, including the sensitivity, response time, recovery time, repeatability, and stability of newly designed $\mathrm{CH}_{4}$ gas sensors.

\section{Experimental}

\section{Fabrications}

Figure 1(a) shows a schematic diagram of the platform with nanowire arrays combined with a pair of external electrodes (labeled as " 1 " and "4") and a pair of internal electrodes (labeled as " 2 " and " 3 ") for $\mathrm{CH}_{4}$ gas sensors. As shown in Fig. 1(b), the spaces are $5 \mu \mathrm{m}$ between the 


\section{Advanced Materials Letters www. vbripress.com/aml}

electrodes "1" and "2", $10 \mu \mathrm{m}$ between electrodes " 2 " and " 3 " and $20 \mu \mathrm{m}$ between electrodes " 3 " and " 4 ". The conductive electrodes " 1 " and " 4 " (the external electrode pair) are connected to a switch, a DC power supply with a step voltage $V_{\mathrm{p}}$, and a high precision resistor $\mathrm{R}$ in series, respectively. One voltmeter $\left(V_{\text {in }}\right)$ is connected to the electrodes " 2 " and " 3 " (the internal electrode pair) and the other voltmeter $\left(V_{\mathrm{ex}}\right)$ is connected in parallel with the resistor in the external electrode circuit. Both voltmeters monitor the voltage variations of the gas sensors formed between the internal electrode pair and the external electrode pair simultaneously.

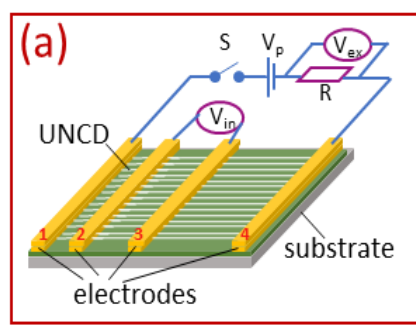

(c)

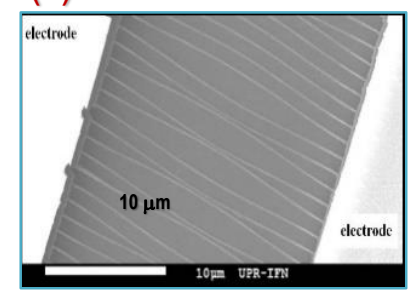

Fig. 1. (a) Schematic diagram of the sensor platform. (b) SEM image of UNCD film (grey) with four Ti/Au (white) electrodes. (c) the magnified SEM image of UNCD nanowires between the two (white) electrodes. (d) Raman spectrum of UNCD recorded with $514 \mathrm{~nm}$ excitation wavelength.

In order to test the operation above room temperature, a thermocouple and tungsten filament are used to control the operating temperature of the sensor. At a given temperature, the sensor output voltage signal is measured as a function of gas concentration.

The sensing material of N-doped UNCD film used in this study was synthesized by the MPCVD technique at Argonne National Laboratory [9]. The film, around 50 100 $\mathrm{nm}$ thick, was deposited on a Si substrate. The microwave frequency for the MPCVD system was $2.45 \mathrm{GHz}$ and the power was around $2 \mathrm{~kW}$. The gas mixture contained $95 \%$ 99\% $\mathrm{Ar}, 0-3 \%$ of $\mathrm{H}_{2}, 1 \%$ of $\mathrm{CH}_{4}$, and other dopant gases, and the chamber pressure remained at around 100 Torr. During synthesis, the substrate temperature was kept around $600{ }^{\circ} \mathrm{C}$.

Unlike polycrystalline diamond (PCD) film or nanocrystalline diamond (NCD) film, the surface roughness of UNCD film is generally very small, around only a few nm (RMS), which doesn't change when the film thickness increases. This is important for nanowire fabrication. In contract to UNCD growth in an Ar-rich environment, for NCD growth in hydrogen-rich condition, hydrogen abstraction takes place to replace each $\mathrm{C}-\mathrm{H}$ bonding of $\mathrm{CH}_{4}$ with $\mathrm{C}-\mathrm{C}$ bonding individually. This process gives the chance for the diamond crystal to grow, leading to a columnar crystal growth with higher surface roughness $[\mathbf{9 , 1 2}]$.

In order to enlarge the contact surface area of the sensor, top-down fabrication techniques were used for achieving UNCD nanowire arrays. The process typically involves design, lithographical patterning, etching, mask deposition, and removal steps. Briefly, $10 / 100 \mathrm{~nm}$ titanium/gold (Ti/Au) or titanium/platinum $(\mathrm{Ti} / \mathrm{Pt})$ layers were deposited on the synthesized UNCD film through a mask to form the four electrodes separated by 5,10 and $20 \mu \mathrm{m}$ gaps between adjacent electrodes. Then the hydrogen silsesquioxane (HSQ) resist was first spincoated at the top, and the nanowire pattern between the electrodes was defined using electron beam lithography, followed by reactive ion etching with the photoresist as an etch mask. After etching nanowires and removal of the photomask, the sample was annealed at $150{ }^{\circ} \mathrm{C}$ for $5 \mathrm{~min}$ in the probe station chamber by a Lake Shore temperature controller. A very detailed description of the MOCVD equipment, synthesis, crystalline structure characterization, and nanowire patterning and fabrication can be found from our previous reports [13].

Fig. 1(c) shows the enlarged SEM image of UNCD nanowire arrays between the two (white) electrodes where the width and length of each diamond wire is around 150 $\mathrm{nm}$ and $20 \mu \mathrm{m}$, respectively. The UNCD sensing composite material was also analyzed by Raman spectroscopy using a triple monochromator with an excitation wavelength of 514 $\mathrm{nm}\left(\mathrm{Ar}^{+}\right.$ion Laser). The microscope focused the laser beam onto the surface of the UNCD. The broadband peak at around $1332 \mathrm{~cm}^{-1}$ confirms the nanodiamond crystallites from $\mathrm{sp}^{3}$ carbon, while the $\mathrm{sp}^{2}$-bonded carbon around 1340$1600 \mathrm{~cm}^{-1}$ (in particular D-band at $1350 \mathrm{~cm}^{-1}$ and G-band at $1580 \mathrm{~cm}^{-1}$ ) are clearly shown in Fig. 1(d). Typically, UNCD films contain the mixtures of $\mathrm{sp} 2 / \mathrm{sp} 3$-bonded carbon (trans-polyacetylene (t-PA) like, and graphite-like $\mathrm{sp}^{2}$-bonded $\mathrm{CH}$ groups and $\mathrm{sp}^{3}$-bonded $\mathrm{CH}_{2}$ groups), with $\mathrm{C}-\mathrm{H}$ vibration characteristics at $1120-1190 \mathrm{~cm}^{-1}$. The peak lower than $1000 \mathrm{~cm}^{-1}$ is not related to the UNCD which could be from the Si substrate [14].

\section{Electrical properties}

To explore the possibility of using the N-doped UNCD nanowire devices, it is essential to understand their electrical characteristics. Fig. 2(a) shows the currentvoltage (I-V) plots of the UNCD nanowires measured by using a pair of internal electrodes at different temperatures. A linear relationship between electrical current and the applied voltage is clearly visible in the low forward current range, indicating there is no additional charge accumulation and very good ohmic contact in the interface between the electrode and the sensing material. However, the reasons why the increase in temperature does not affect the conductivity of N-doped UNCD nanowires, in this case, has not yet been fully understood. The estimated device resistance between the internal electrodes $R_{d}=\Delta V / \Delta I \approx 33$ $\mathrm{k} \Omega$ shows the high conductivity of the N-doped UNCD 


\section{Advanced Materials Letters www. vbripress.com/aml}

nanowires. The I-V properties of the UNCD nanowires were also characterized by using a pair of external electrodes as shown in Fig. 2(b), which are almost linear, giving estimated device resistances of $188 \mathrm{k} \Omega$ at $250{ }^{\circ} \mathrm{C}$, $219 \mathrm{k} \Omega$ at $100{ }^{\circ} \mathrm{C}$ and $350 \mathrm{k} \Omega$ at room temperature between the external electrodes. Using a simple wire model for resistance $R_{d}=\rho l / A$ where $l$ is the length and $A$ is its crosssectional area, the different resistivities $\rho$ can be estimated. This is attributed to the high incorporation level of nitrogen that affects the original electron transport. The resistance of the UNCD decreases with the temperature.

It has been found that the doping content and doping element, in general, significantly affect UNCD properties. For example, boron-doped UNCD is an excellent active material for deep UV photo-detectors, [15] whereas nitrogen-doped UNCD is a good material for gas sensing device but poor response to deep UV radiation. The present work focuses on the UNCD gas sensing device.
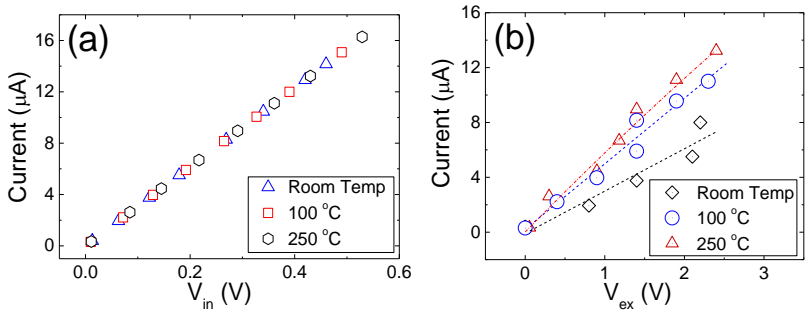

Fig. 2. Electrical properties of UNCD characterized in the low forward current range at different temperatures by using (a) an internal pair and (b) external pair of electrodes.

\section{Prototypic $\mathrm{CH}_{4}$ gas sensors}

The circuit used for the prototypic gas sensor test is shown in Fig. 1(a). This simple but useful electrical circuit has been widely used for oxide semiconductor gas and humidity sensors [16-18].

The variation of the voltage output of the fabricated sensor is caused by the reaction of incoming gaseous molecules or targeted gas with the chemisorbed oxygen at the UNCD grain boundaries that change the nanowires' conductivity. The UNCD nanowire has the ideal structure with a large number of grains and boundary surfaces, as well as a large surface area to volume ratio. The electrical properties of the nanowire surface and grain boundaries are affected by the adsorption and desorption of the gaseous molecules. As a result, negative charge carriers are added to the material and hence the resistance decreases [19]:

$$
\mathrm{CH}_{4}+4 \mathrm{O}_{\text {adsorption }}^{-} \rightarrow \mathrm{CO}_{2(\text { air) }}+2 \mathrm{H}_{2} \mathrm{O}+4 \mathrm{e}^{-}
$$

Therefore, we can detect the $\mathrm{CH}_{4}$ gas concentration by measuring the conductivity change of the UNCD nanowires. From the measurement of variation of voltage ( $\left.V_{e x}\right)$ across the precision resistor $R=1.0 \mathrm{k} \Omega$, the resistance of the sensor can be obtained by $R_{d}=\left(V_{p}-V_{e x}\right) R / V_{e x}$ where the power supply voltage $V_{p}=12 \mathrm{~V}$. Necessary calibrations of the sensor were conducted at the characterization chamber (see Fig. 3) [20]. It includes a plasma beam source for the treatment of the surface of the sensor device, the gas flow meter and controller, a precision pressure gauge, and a mass spectrometer.

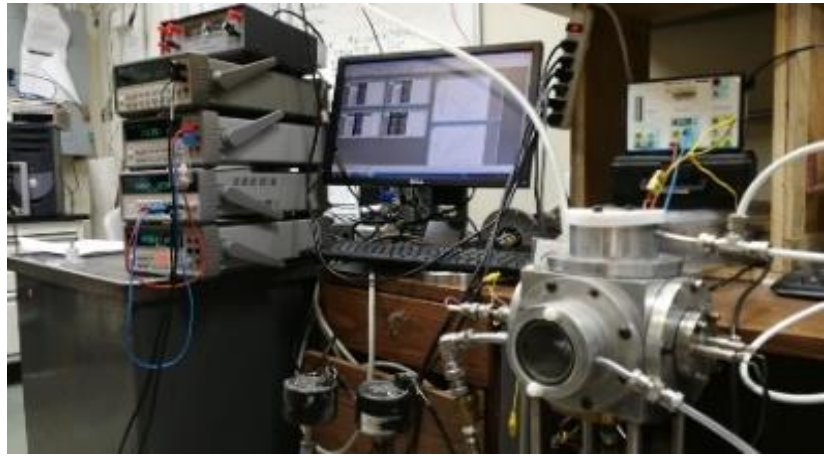

Fig. 3. Photograph of experimental set up for characterization of the newly fabricated gas sensors.
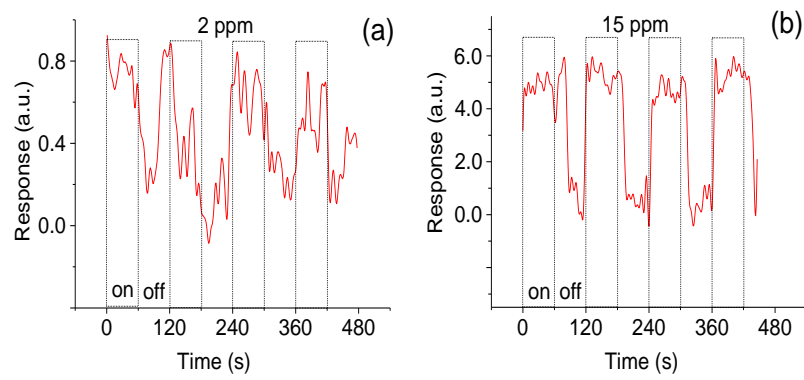

Fig. 4. Typical responsivities from the internal electrode pair of the nanowire N-doped UNCD gas sensor to the "on" and "off" of the methane gas at the concentration of (a) $2 \mathrm{ppm}$ and (b) $15 \mathrm{ppm}$.

\section{Results and discussion}

The characterizations of the fabricated sensors include repeatability and stability, which are the most important parameters for a gas sensor, as well as sensitivity/ resistance, the response time, and the recovery time. Fig. 4(a) shows the typical responsivity when the nanowire diamond gas sensor was cycled with a period of 120 seconds between "switch on" and "switch off" for the inlet valve for the methane gas. The measurements are based on the pair of internal electrodes. The concentration of the gas molecule is 2 ppm for the results shown in Fig. 4(a) and 15 ppm for Fig. 4(b). The sensor shows good repeatability and stability. The changes in the conductivity can be attributed to the adsorption of methane molecules. The response and recovery times of the sensors are only a few seconds as shown in Fig. 4(a).

Fig. 4(b) depicts the response of the fabricated gas sensor tested at methane concentrations of $15 \mathrm{ppm}$, where the gas flow rate was controlled at $1.5 \mathrm{sccm}$. The measurements are performed at room temperature. The period for the cycle is manually controlled at 120 seconds. The charge of the electrical resistance of the nanowires is found upon exposure to methane gas.

As a comparison, the fabricated sensor is also characterized by using a pair of external electrodes. Stability or repeatability features obtained from the cycled test are shown in Fig. 5(a) where the concentration of methane gas is $15 \mathrm{ppm}$. One can easily find that when the sensor is exposed to the methane gas, the resistance of the 


\section{Advanced Materials Letters www. vbripress.com/aml}

sensor promptly decreased and then reached a relatively stable value. When the targeted gas is removed, the resistance abruptly increased and then gradually reached a relatively stable value.

It is noted that the responsivities of the internal electrode pair do not behave so well as the external electrode pair, because the present device consists of UNCD nanowires with ohmic contacts. When exposed to the targeted gas, the change of the conductance of the UNCD nanowires relies on the gas molecules adsorbed on to the surface of the active layer. Therefore, a larger exposure area of the active layer should have a better responsivity.
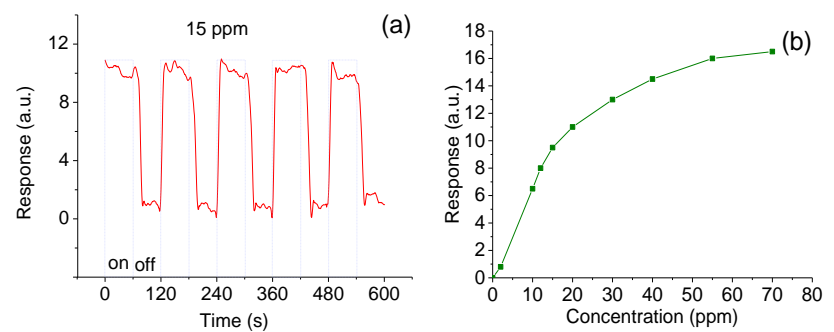

Fig. 5. Responsivities from the pair of the external electrodes to the methane gas with (a) a concentration of $15 \mathrm{ppm}$ and (b) continuous increase of the concentration from $0-70 \mathrm{ppm}$, measured at room temperature.

As shown in Fig. 5(a), good baseline stability is always visible for the fabricated sensor operating at room temperature. When the methane gas concentration continuously increases from 0 to $70 \mathrm{ppm}$, the trend of the relative sensitivity of the fabricated sensor is shown in Fig. 5(b). According to the sensitivity defined as $S=$ $\left(\mathrm{R}_{\mathrm{CH} 4}-\mathrm{R}_{\text {air }}\right) / \mathrm{R}_{\text {air }} \times 100 \%$, where $\mathrm{R}_{\mathrm{CH} 4}$ is the sensor resistivity measured in the presence of $\mathrm{CH}_{4}$, and $\mathrm{R}_{\text {air }}$ is the initial sensor resistivity in the absence of the target gas $\mathrm{CH}_{4}$, the obtained relative sensitivity is 2,8 , and $16 \%$ at 2,12 , and $55 \mathrm{ppm}$, respectively, when measured at room temperature.

In general, at high concentrations of methane gas, the output signal of the fabricated gas sensor is strong. For example, at $15 \mathrm{ppm}$ the sensor output is almost 6 times larger than that at 2 ppm with the pair of internal electrodes. Furthermore, the external electrodes-based sensor has wellshaped edges in its output signal, indicating a fast response time.

In fact, the response-recovery time of the sensor depends on many parameters such as the nature of samples, concentration, operating temperature, and electrode arrangement. In the case of low concentration of the targeted gas, the time response and recovery are delayed, which can be explained by a lack of molecules participating in the reaction with the UNCD nanoparticles, yielding more time required for reaching the balance of the reaction between the sensing material and the targeted molecular gas.

In contrast, in the high concentration case, a much shorter period is needed to reach equilibrium in the reaction. Consequently, a fast time response can be obtained. It is also noted that each response profile from the fabricated sensor has a sharp edge as shown in Fig. 5(a), which strongly suggests that the UNCD based gas sensor has a very quick responsivity. This is in good agreement with the data shown in Fig. 4(b).

In order to obtain the precise values of the time responsivity, dynamic measurements are carried out as shown in Fig. 6(a) where the red curve is related to the response time and the black curve is related to recovery time. Correspondingly, the response time of 3 seconds and the recovery time of 9 seconds are obtained. The definition is based on the time duration from $10 \%$ to $90 \%$ of the full response of the sensor. Clearly, the obtained time response value is much shorter than that of regular sensors which are usually about $100 \mathrm{~s}$ for the response time and more than 200 $\mathrm{s}$ for the recovery time $[\mathbf{2 1}]$.
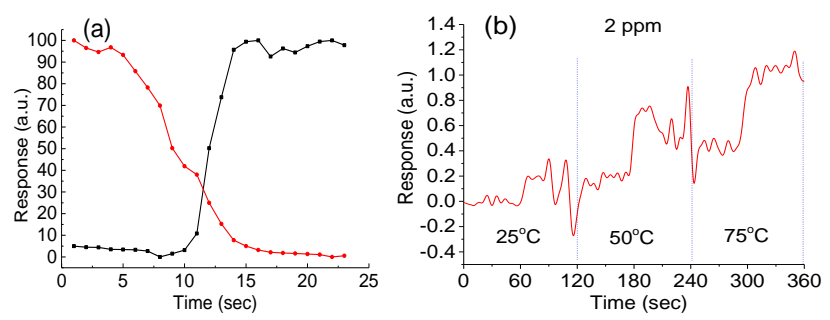

Fig. 6. Sensor response to methane gas at concentrations of (a) $15 \mathrm{ppm}$ at room temperature, and (b) $2 \mathrm{ppm}$ at the operating temperature of 25,50 , and $75^{\circ} \mathrm{C}$.

It is expected that the real sensing response time and recovery time for the present gas sensor might be shorter. This is because of how the time delay in turning on or off the valves of the gas inlet and outlet, as well as a low pumping capacity $\left(7 \mathrm{~m}^{3} / \mathrm{h}\right)$, would affect the measured results.

The operating temperature has a great influence on the properties of the UNCD nanowire arrays-based sensor. Fig. 6(b) shows the temperature effect on the responses of the sensor when exposed to the targeted gas. At room temperature $\left(25^{\circ} \mathrm{C}\right)$ when the fabricated sensor is exposed to the $2 \mathrm{ppm}$ methane gas. At an operating temperature of $50{ }^{\circ} \mathrm{C}$ where the $2 \mathrm{ppm}$ concentration of the methane gas remained unchanged, the output of the sensor increased and then reached a stable value. However, no obvious improvement in response time is observed. A similar phenomenon was also observed at an operating temperature of $75^{\circ} \mathrm{C}$ as shown in Fig. 6(b) but the output of the sensor increased. The probable reason for no outstanding rise time is because of time delay during adjustment of the gas flow rate to a specific rate of $1.5 \mathrm{sccm}$.

It is also found that operating UNCD nanowire arraysbased sensors at high temperatures would cause instability in its response and output, resulting in poor baseline stability. This phenomenon is, in fact, related to defects in the interface. In the case of $\mathrm{Ti}$ and $\mathrm{Al}$, the carbide that formed during deposition and/or earlier stages of annealing limited the diffusion of these defects. As a result, these 


\section{Advanced Materials Letters www. vbripress.com/aml}

contacts on the diamond surface retained their ohmic character even after the annealing. If the defect is the dominant mechanism governing the electrical properties of the metal-diamond interface, then ohmic contacts formed on the diamond surface with carbide forming reactions are accompanied by defect formation at the interface. The contacts suffer from high temperature stability problems since those defects are not thermally stable [22]. More studies are necessary in order to establish the reliability of metal contacts for diamond-based sensing devices operating at high temperatures.

Finally, several sources of error or uncertainty have been observed during measurements and estimations. For example, these error sources were from the gas flow rate instability that might affect target gas concentration, and electronic signal noises. Some errors could also occur due to the presence of temperature gradients, drafts, poor thermal contact, calibration drifts, etc. After making a comparison of all data from repeated experimental data, we could conclude that the relative error is less than $15 \%$ in the presented results.

\section{Conclusion}

The N-doped UNCD nanowires have been fabricated with metal contact as electrodes layered over nanowires. High performance methane gas sensors have been designed and tested. Both the internal and external electrode effects and temperature effects on the sensing properties have been investigated. Analyses of the experimental data clearly indicated the fabricated nanowire diamond sensor has excellent features such as good repeatability and stability at room temperature. The response time of 3 seconds and the recovery time of 9 seconds are obtained. To the best of our knowledge, the fabricated UNCD quasi-one-dimensional nanowires have achieved the highest sensitivity and fastest response and recovery times at room temperature of all semiconductor based methane gas sensors reported so far, although the sensor performance could be further improved by optimizing the doping concentration and nanowire structure.

We conclude that the high concentration of the methane gas results in a strong signal output from the sensor but the output signal strength is not linearly related to the targeted gas concentrations. The relative sensitivity of the fabricated sensor is high at low concentrations. Too high concentrations of the methane gas would cause output saturation from the sensor. It is also found that the response or sensitivity of the sensor to the targeted gas would be enhanced at a slightly higher operating temperature. However, no obvious improvement in response time is observed. Our experimental result shows the nitrogendoped UNCD is an excellent material candidate for novel gas sensor applications.

\section{Acknowledgements}

This work is financially supported by NSF-CREST Center for Innovation, Research and Education in Environmental Nanotechnology (CIRE2N) Grant Number HRD-1736093. The authors also would like to thank Drs. Anirudha Sumant, Ralu Divan, Leo Ocola and Daniel Rosemann from Argonne National Lab for the help of UNCD nanowire fabrication. Use of the Center for Nanoscale Materials was supported by the US Department of Energy, Office of Science, Office of Basic Energy Sciences, under Contract No. DE-AC02-06CH11357. AFZ acknowledges the receipt of the 2017-2018 IUP Senate Fellowship grant and the partial support of this work by the NSF CMS-1725557.

\section{Keywords}

Nitrogen doping, ultrananocrystalline diamond (UNCD), nanowire, gas sensor, $\mathrm{CH}_{4}$

Received: 13 August 2019

Revised: 17 December 2019

Accepted: 23 December 2019

\section{References}

1. Wang, Q.; Qu, S. L.; Fu, S. Y.; Liu, W. J.; Li, J. J.; Gu, C. Z.; J. Appl. Phys., 2007, 102, 103714.

2. Helwig, A.; Muller, G.; Garrido, J. A.; Eickhoff, M.; Sens. Actuators, B: Chemical, 2008, 133, 156.

3. Gurbuz, Y.; Kang, W. P.; Devidson, J. L.; Kerns, D. V.; Sens. Actuators, B: Chemical, 2004, 99, 207.

4. Peng, X.; Chu, J.; Wang, L.; Duan, S.; Feng, P.; Sens. Actuators, B: Chemical, 2017, 241, 383.

5. Gurbuza, Y.; Kang, W. P.; Davidson, J. L.; Kerns, D. V.; Sens. Actuators, B: Chemical, 2004, 99, 207.

6. Das, S.; Jana, S.; De, D.; Gangopadhyay, U.; Garain, S.; Ray, S.; Mondal, A.; Ghosh, P.; A Novel Room Temperature Ammonia Gas Sensor Based on Diamond-Like Nanocomposite/ c-Silicon Heterojunction in Physics of Semiconductor Devices. Environmental Science and Engineering. Jain, V.; Verma. A. (Editors); Springer, Cham, 2014, pp 479-482.

7. Davydova, M.; Kulha, P.; Laposa, A.; Hruska, K.; Demo, P.; Kromka, A.; Beilstein J. Nanotechnol., 2014, 5, 2339.

8. Auciello, O.; Science and Tecnnology of Ultrananocrystalline Diamond Film Based MEMS and NEMS Devicess and Systems in Ultrananocrystalline Diamond: Syntheses, Properties, and Applications (Second Edition); Shenderova, O. A. and Gruen, D. M. (Editors); Elsevier, 2012; pp 383-420.

9. Auciello O.; Sumant, A. V.; Diamond Relat. Mater., 2010, 19, 699.

10. Chen X.; Huang Z.; Li J.; Wu C.; Wang Z., Cui Y.; Vacuum, 2018, 154, 120.

11. Feng, P.; Wang, X.; Aldalbahi, A.; Zhou, A. F.; Appl. Phys. Lett., 2015, 107, 233103.

12. Wang, X.; Synthesis, Fabrication, Characterization and Application of Ultrananocrystalline Diamond Micro- and Nanostructures. Doctoral dissertation, University of Puerto Rico: San Juan, Puerto Rico, 2012.

13. Wang, X.; Ocola, L. E.; Divan, R. S.; Sumant, A. V.; Nanotechnology, 2012, 23, 075301.

14. Teng, C.; Song, S.; Sung, C.; Lin, C.; Journal of Nanomaterials, 2009, 2009, 621208 .

15. Zhou, A. F.; Velazquez, R.; Wang, X.; Feng, P.; ACS Appl. Mater Interfaces, 2019, 11, 38068.

16. Mandal, S.; Naud, C.; Williams, O. A.; Bustarret, E.; Omnes, F.; Rodiere, P.; Meunier, T.; Saminadayar, L.; Christopher, B.; Nanotechnology, 2010, 21, 195303.

17. Peng, X.; Yang, B.; Chu, J.; Feng, P.; Sens. Actuators, B: Chemical, 2012, 174, 258

18. Chu, J.; Peng, X.; Feng, P.; Sheng, Y.; Zhang, J.; Sens. Actuators, B. Chemical, 2013, 178, 508.

19. Basu, S.; Basu, P. K.; Journal of Sensors 2009, 2009, 861968.

20. Chu, J.; Peng, X.; Sajjad, M.; Yang, B.; Feng, P.; Thin Solid Films, 2012, 520, 3493.

21. Feng, P.; Zhang, H.; Peng, X.; Sajjad, M.; Chu, J.; Review of Scientific Instruments, 2011, 82, 043303.

22. Tachibana T.; Glass J. T.; Electrical Contacts to Diamond in Diamond: Electronic Properties and Applications; Pan, L. S.; Kania, D. R. (Editors); The Kluwer International Series in Engineering and Computer Science (Electronic Materials: Science and Technology), Springer, 1995, pp 319-348. 